of (1) can be represented as a power series, a contour integral, or as a differential operator. For example, from a formal use of Taylor's theorem it follows that

$$
F(z+y, \alpha)=\sum_{0}^{\infty} y^{n} F(z, \alpha+n) / n !
$$

This formula gives directly a generating function for the sequence $F(z, \alpha+n) / n !, n=0,1,2, \cdots$, while for $z=0$ it gives a power series expansion of $F(y, \alpha)$.

As a result of these techniques the author is able to handle problems which could be treated only with difficulty by classical methods. For example, express the Laguerre polynomials in terms of the Legendre polynomials. The scope of the method and the ingenuity of the author are illustrated by the derivation of new, complicated formulas involving the special functions.

An essential point in the study of equation (1) is the proof of the following theorem: Given a bounded sequence $\phi(\alpha+n), n=0,1,2, \cdots$, there exists one and only one solution of (1) such that

$$
F\left(z_{0}, \alpha+n\right)=\phi(\alpha+n), \quad n=0,1,2, \cdots .
$$

This theorem is obtained as a special case of an existence theorem for a general vector difference-differential equation which the author proves. Once the uniqueness is known, it is easy to justify the formal applications such as those in (2).

The author concludes his monograph with some still unsolved questions. One such question is this:

What are the conditions to ensure the existence of a unique solution of the equation

$$
\frac{\partial F(z, \alpha)}{\partial z}=F(z, \alpha-1)
$$

when $\alpha=\alpha_{0}+n, n=0,1,2, \cdots$ ? If the answer to this were known, some important identities involving number-theoretic functions would be immediate consequences of (3).

\title{
BERNARD FRIEDMAN
}

Non-linear problems in mechanics of continua. (Proceedings of Symposia in Applied Mathematics, vol. 1.) New York, American Mathematical Society, $1949.8+219$ pp. $\$ 5.25$.

This volume contains papers presented at the First Symposium in Applied Mathematics, held at Brown University, August 2-4, 1947. 
The subject of the symposium was Non-linear problems in mechanics of continua. The content of the volume falls into two groups: the first (157 pages, sixteen papers) is concerned with fluid mechanics, the second (60 pages, eight papers) with elasticity and plasticity. The articles differ widely in both length and character.

The papers of the first group offer an instructive sample of the great variety of problems considered today in the above described direction. Several articles deal with the problems of so-called free boundaries. The first article in the book is a comprehensive and interesting paper by A. Weinstein entitled Non-linear problems in the theory of fluid motion. There is a brief abstract of work completed and in progress on recent developments in free boundary theory by G. Birkhoff. Non-linear free boundary problems arise likewise in the consideration of wave motion in water with a free surface. J. J. Stoker presents a study, The breaking of waves in shallow water, with emphasis on the mathematical analogy with shocks in gas dynamics. Shock problems form the subject matter of a few other articles. An extensive paper which reviews some less accessible material while also presenting new results and viewpoints has the title $O n$ shock phenomena: interaction of shock waves in gases written by $\mathrm{H}$. Polachek and R. J. Seeger. A paper Theory of the propagation of shock waves from cylindrical charges of explosives is presented by S. R. Brinkley, Jr. and J. G. Kirkwood. The general problem of the integration of the equations of gas dynamics is studied in a number of papers under different aspects. S. Bergman presents a condensed review of his important and extensive work on Operator methods in the theory of compressible fluid flow. L. Bers, in an article An existence theorem in two-dimensional gas dynamics gives a complete existence theorem for the flow around a profile under the assumption of Chaplygin's well known linear approximation to the adiabatic relation. If this relation is approximated by a polygon rather than by a single straight line, a possibly efficient method for the numerical solution of flow problems is obtained in H. Poritsky's paper Polygonal approximation methods in the hodograph plane. The interesting article of L. Opatowski extends some results of Volterra and Levi-Civita on incompressible flow to compressible flow problems. A. H. Taub's paper on a variational principle for perfect compressible fluids may likewise be mentioned in this connection. A contribution to the integration theory in case of three-dimensional flow problems is offered in the article of $\mathrm{N}$. Coburn and C. L. Dolph, The method of characteristics in the three-dimensional stationary supersonic flow of a compressible gas. Problems of boundary layer theory (incompressible flow) are considered in the papers by 
L. Lees, Stability of the laminar boundary layer in a compressible fluid, and by R. Sears, The boundary layer of yawed cylinders. H. W. Emmons proposes to study turbulent flow by directly attacking the Navier-Stokes equations through a method of finite-differenceintegration in a paper The numerical solution of the turbulence problem. A very interesting contribution to the statistical theory of turbulence, so much discussed today, is offered in C. C. Lin's article Remark on the spectrum of turbulence. M. Kuo comments briefly on the stability of a flow in the neighborhood of the sonic point $(M=1)$.

It is of course impossible to do justice in a brief review to numerous articles, widely different in scope and character. What is termed "interesting" or "important" depends rather more on the reviewer's interests and training than on objective standards. In fact no evaluation can be attempted. Rather the aim is to assist the reader in forming some opinion as to whether the volume as a whole or one or more of the articles will be of interest to him.

The second group starts with F. D. Murnaghan's basic contribution Foundations of the theory of elasticity which studies the problem of non-infinitesimal displacements. A group of papers may be singled out that deal with large deflection of plates while small strains are still considered. There is E. Reissner's very competent paper On finite deflections of circular plates, S. Levy's paper Large deflection theory for rectangular plates, and the suggestive paper by K. Friedrichs on the problem of buckling, The edge effect in bending and buckling with large deflections. A non-linear strain-stress relation is assumed by W. Kaplan and dealt with by a numerical approach in the article Numerical methods in the solution of problems of non-linear elasticity. G. F. Carrier in the article On dynamic structural stability attempts to extend the stability theory of structures to the case of dynamic loads. There are only two papers on plasticity, one an abstract of W. Prager's stimulating paper on discontinuous solutions in plasticity theory, which appeared in full in the Courant Anniversary volume, and the other D. C. Drucker's paper Stressstrain relations for strain hardening materials: discussion and proposed experiments where the so-called "deformation theories" of plasticity versus "plastic flow" theories are examined, the latter appearing preferable.

On the whole, the volume offers an important cross section of certain aspects of work in applied mathematics.

Hilda Geiringer 